\title{
A case of near-sudden unexpected death in epilepsy due to ventricular fibrillation
}

This article was published in the following Dove Press journal:

Open Access Emergency Medicine

\section{Myung $\mathrm{Ha} \mathrm{Seo}$ \\ Won Young Sung (1D}

Department of Emergency Medicine, Eulji University Hospital, Eulji University College of Medicine, Daejeon, Republic of Korea
Correspondence: Won Young Sung Department of Emergency Medicine, Eulji University Hospital, Eulji University College of Medicine, 95, Dunsanseo-ro, Seo-gu, Daejeon 35233, Republic of Korea

Tel +82 42 6II 3256

Fax +82426113880

Email sage77@hanmail.net

\begin{abstract}
Sudden unexpected death in epilepsy (SUDEP) refers to the sudden and unexpected death of an epileptic patient with no other health issues, during normal activity, and for whom no other particular cause of death can be found. The exact cause of SUDEP has not been established yet; however, it is assumed to be caused by multiple organ failure involving the respiratory and cardiovascular systems. Some of the known risk factors are generalized tonic-clonic seizure, frequent epileptic seizure, early onset of epilepsy, long duration of seizure, nocturnal seizure, and combined therapy with antiepileptics. A number of seizure-related cardiac arrhythmia cases have been reported. Arrhythmias are mostly benign tachycardia or bradycardia, and ventricular fibrillation (VF) or asystole is very rare. It is considered that fatal cardiac arrhythmia is a cause of SUDEP. Here, we describe the case of a near-SUDEP patient who was successfully revived without complications by immediate defibrillation with an automated external defibrillator and cardiopulmonary resuscitation, although VF occurred after a convulsive seizure. Based on our experience, when treating a patient with an epileptic seizure, one should always keep in mind the possibility of SUDEP as a seizure-induced emergency situation involving fatal arrhythmia and cardiac arrest, even in young healthy adults.
\end{abstract}

Keywords: seizure, cardiac arrhythmia, sudden unexpected death in epilepsy, ventricular fibrillation

\section{Introduction}

Sudden unexpected death in epilepsy (SUDEP) refers to a sudden and unexplained death in a patient with epilepsy that is not attributable to trauma, drowning, status epilepticus, or another known cause but for which there is often evidence of an associated seizure. ${ }^{1}$ The etiology of SUDEP remains unknown. Multiple pathophysiologic factors are thought to be involved with respiratory and cardiac dysfunctions being the main ones.

Seizure-related cardiac arrhythmias are frequently reported and have been implicated as potential mechanisms underlying SUDEP. ${ }^{2}$ The most common periictal arrhythmia is sinus tachycardia, which accompanies $90 \%$ of all seizures. Bradycardia and asystole are uncommon and occur in only about $0.5 \%$ of seizures. ${ }^{3}$ Post-ictal arrhythmias, including asystole, atrioventricular (AV) block, and the less prevalent atrial fibrillation (AF) and ventricular fibrillation (VF), usually occur after a convulsive seizure and are frequently associated with SUDEP. ${ }^{2}$ To date, only in a few cases of VF arising from a seizure, near-SUDEP has been reported. ${ }^{4-7}$

Here, we report a rare case of VF that was been successfully managed using defibrillation with an automatic external defibrillator (AED) and cardiopulmonary resuscitation (CPR) after a convulsive seizure. 


\section{Case presentation}

A 48-year-old female patient presented to the emergency department (ED) after CPR due to VF that occurred after a seizure. Four years prior, she presented to other hospital ED and discharged after observation for a condition where her arms were raised and her limbs were stretched continually for approximately $1 \mathrm{~min}$ while sleeping after an episode of alcohol intake. She did not undergo any particular tests and neither was she administered any drugs, under the assumption that these symptoms were temporary and had been caused by alcohol intake. Three years prior, a similar episode occurred during sleep, for which some medical tests were conducted such as electrocardiogram (ECG), brain MRI, and electroencephalogram (EEG), but no conclusive results were reported. Drugs were also prescribed but not taken by the patient. One and a half year prior, the patient experienced another relapse again during sleep at dawn. According to the statement given by her husband who witnessed the episode at the time, for approximately $1 \mathrm{~min}$ while sleeping, the patient groaned with her mouth shut tightly, her arms raised, limbs stretched, and did not respond when called. Subsequently, her body collapsed, she stopped breathing for approximately $30-40 \mathrm{~s}$, and then she breathed in a concentrated manner. Urination and post-ictal confusion were also noted. The patient had no family history of epilepsy or no medical history of febrile convulsion in the past, and only reported of the inability to sleep well and waking up frequently due to a burning sensation in the foot. In the previous hospital, the patient underwent videoEEG and polysomnography in an epilepsy monitoring unit (EMU) for two days to investigate the cause under the putative diagnosis of restless legs syndrome and nocturnal bilateral tonic seizure - frontal lobe epilepsy. Based on EEG findings, there was no inter-ictal epileptiform discharge and an intermittent slow wave was observed in the right frontotemporal area, but it was equivocal, and therefore, no clear abnormality was reported. Subsequently, administration of pramipexole and pregabalin was started to control the rare nocturnal seizures and symptoms of the restless legs syndrome under the putative diagnosis of restless legs syndrome and unknown etiologic epilepsy - bilateral tonic seizure.

On the day before current admission, her epileptic seizure relapsed during sleep but she recovered consciousness immediately; thus, she did not come to the hospital. In the evening of the admission day, while conversing with her children, she suddenly had a general tonic-clonic type seizure for 2-3 mins and emergency medical services (EMS) were informed as she did not recover consciousness immediately after the seizure like in previous episodes and her breathing was almost negligible. Following the instructions of the EMS center, her family started CPR. After the paramedics arrived, spontaneous circulation was restored after CPR and 2 times of defibrillation as VF was indicated by the AED findings (Figure 1). At the time of ED arrival, the patient was stupor and her vital signs and oxygen saturation were normal with a blood pressure of $121 / 82 \mathrm{mmHg}$, pulse rate of 80 beats per minute, respiratory rate of 16 breathes per minute, and body temperature of $36.2^{\circ} \mathrm{C}$. ECG performed immediately after arrival showed a sinus rhythm with a heart rate of 78 beats per minute and QTc duration of $401 \mathrm{~ms}$ (Figure 2). The initial arterial blood gas analysis (ABGA) showed a $\mathrm{pH}$ of 7.35, $\mathrm{PCO}_{2} 21 \mathrm{mmHg}, \mathrm{PO}_{2} 134 \mathrm{mmHg}, \mathrm{HCO}_{3} 11.6 \mathrm{mEq} / \mathrm{L}$, and a lactate level of $6.1 \mathrm{mg} / \mathrm{dL}$. Approximately $10 \mathrm{~min}$ after arrival, she regained consciousness but Todd's paralysis in the right limbs and global aphasia were shown. The repeat ABGA showed that all values had recovered to normal levels. All other blood test results were normal, including electrolyte and myocardial enzyme levels. Levetiracetam was administered intravenously in the ED. Brain diffusion MRI showed no abnormality. Thereafter, the patient was admitted to the cardiology intensive care unit (ICU) and received a full cardiologic work-up. After admission to the ICU, her consciousness was maintained in an alert state, the paralysis in the right limbs and aphasia resolved completely, and the EEG showed normal results. Transthoracic echocardiography performed on the day after admission showed that the left ventricle ejection fraction (LVEF) was reduced to $44 \%$, and left ventricle global hypokinesia and moderate systolic dysfunction were observed. After that, consecutively performed coronary angiography (CAG), spasm provocation test, cardiac MRI, and 24-hr Holter monitoring showed normal results. For the purpose of preventing sudden cardiac death (SCD), the insertion of an implantable cardiac defibrillator (ICD) was recommended. The patient was discharged without any complications 6 days after the procedure. Currently, levetiracetam administration is being maintained by the neurology department and her condition is being periodically followed by the cardiology department under the diagnosis of aborted SCD due to VF and heart failure with midrange ejection fraction. 

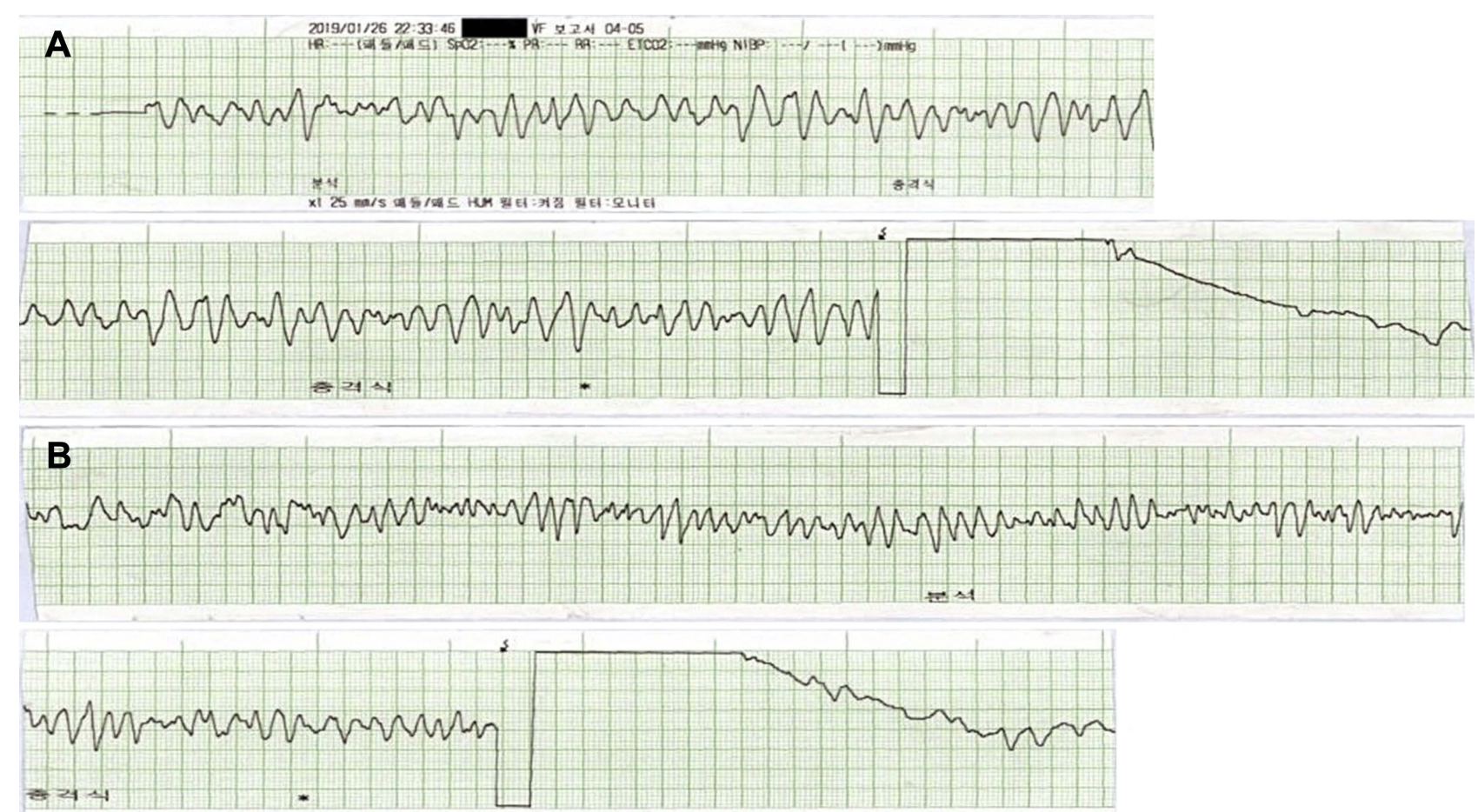

Figure I Prehospital electrocardiographic recordings of the patient. The recordings showed the first (A) and second (B) defibrillation after detection of ventricular fibrillation by automatic external defibrillator.

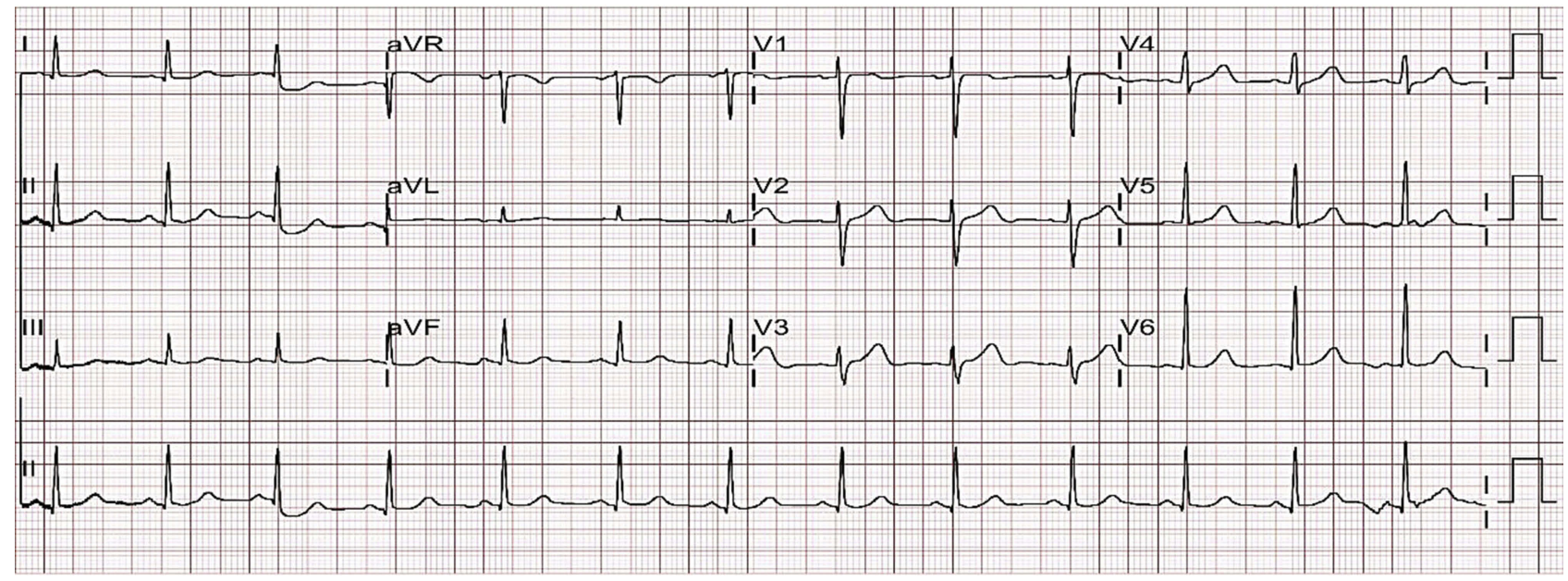

Figure 2 Initial electrocardiography performed in the emergency department showed sinus rhythm with a heart rate of 78 beats per minute and normal range corrected QT interval.

\section{Discussion}

Near-SUDEP is defined as more than 1-hr survival from cardiopulmonary arrest after a seizure that has no identified structural cause after investigation. ${ }^{8}$

A few cases of VF arising from seizure have been reported. ${ }^{4-7}$ One patient had a basal first degree AV block, but had no major risk factors for VF, as indicated by normal CAG and electrophysiology study findings, except focal LV wall hypokinesia. ${ }^{4}$ In another case report, the patient had normal cardiac workup results (normal basal ECG, transthoracic echocardiography, and cardiac MRI). ${ }^{5}$ In the third case report, the patient had VF after a seizure. ${ }^{6}$ The authors argued that the evidence was not conclusive regarding underlying cardiovascular risk factors and basal ECG abnormalities being attributable for the increase in the risk of SUDEP, but the patient was 
considered to be at high risk for primary VF and SCD at any time due to previous structural heart diseases, such as myocardial infarction, dilated cardiomyopathy, and AF. In a previous report on anesthesia induction for chronic epilepsy surgery, VF occurred after the insertion of a guidewire through the right subclavian vein, and the patient recovered after CPR. ${ }^{7}$ However, it was unclear whether VF was induced by the guidewire and whether a seizure occurred or not, and there were no cardiac workup results to rule out structural heart disease. In the last two cases, it was not clear if the patients satisfied the near-SUDEP definition. In the present case, the patient had a convulsive seizure with respiratory failure, and her caregiver immediately started CPR. After the paramedics arrived, defibrillation was successfully performed using an AED and spontaneous circulation was restored. Echocardiography showed mid-range HF as LVEF was reduced, but a number of cardiac examinations could not detect a clear structural heart disease that could be the cause of VF; thus, we think that it was a case of seizure-induced VF and nearSUDEP.

Many previous studies have suggested that the cause of SUDEP is multifactorial and incorporates factors such as respiratory, cardiac, cerebral, and autonomic dysfunction. ${ }^{9}$ Impaired respiration (respiratory arrest, labored breathing, suffocation in prone position, and laryngeal spasm) as a cause of death is supported by data from multiple studies in animals and evidence from most witnessed and recorded instances of SUDEP. ${ }^{1}$ Seizures and the post-ictal state can affect the brain stem respiratory centers. Central and obstructive apnea are both observed during a seizure. Respiration depends on brain stem activity, and thus, prolonged suppression stops respiration. ${ }^{1}$ The basis for the airway obstruction was demonstrated to be seizureinduced laryngospasm. ${ }^{10}$ Cardiac events are considered to be likely culprits in some SUDEP cases. ${ }^{1}$ Tachyarrhythmia, bradyarrhythmia, asystole, AF, supraventricular tachycardia, and $\mathrm{AV}$ blocks are commonly reported during ictal, inter-ictal, and post-ictal phases in patients with epilepsy. However, the degree to which these rhythm disturbances contribute to SUDEP is unclear. ${ }^{11}$ Lende et $\mathrm{al}^{2}$ reviewed 178 case reports and series, and no deaths were reported suggesting that ictal arrhythmias are self-limiting. In contrast, post-ictal arrhythmias (asystole: 13 cases, AV block: 2 cases, and the less prevalent AF: 14 cases and VF: 3 cases) usually occurred after a convulsive seizure and were frequently associated with (near-) SUDEP.
The precise mechanisms underlying SUDEP and VF arising from a seizure are not clear. Cardiac fibrosis and myocyte hypertrophy, increased QT interval lengths and dispersion (channelopathies and long QT interval syndrome), evidence for increased sympathetic tone, and decreased parasympathetic tone all make investigating whether VF may be a cause of SUDEP. ${ }^{12,13}$ In previous studies, the most common pathology involving the cardiac system was fibrosis of the myocardium, but a recent study did not report elevated prevalence of cardiac fibrosis among SUDEP cases. ${ }^{14}$ Hypoxia could lower the threshold for cardiac arrhythmias during the seizure, especially in patients with channelopathies affecting both brain and cardiac tissue (long QT-syndrome type 2). ${ }^{1}$ In one EMU study, potentially high-risk cardiac arrhythmias were associated with the duration of peri-ictal hypoxia. ${ }^{15}$ Each seizure was able to increase parasympathetic activity and sympathetic tone by about 10 -fold. Increased autonomic nervous system activity resulted in changes in the heart rate and rhythm, which could be brady or tachyarrhythmia. ${ }^{10}$ Increased sympathetic activity and catecholamine rush have also been implicated as triggers for $\mathrm{VF}^{2}$ In animal model studies, authors suspected that periods of severe bradyarrhythmia or asystole might disable some regions of the intrinsic conduction system or ventricular myocardium thereby disrupting the normal sequential activation of myocytes and favoring VF. ${ }^{10}$ Badheka et $\mathrm{al}^{16}$ investigated the outcomes of six epileptic survivors from SCD using the antiarrhythmics versus ICD study database. In that study, epilepsy was a significant predictor of recurrent, life-threatening ventricular arrhythmias and cardiac death. To this day, no large studies of epilepsy patients with an ICD have demonstrated a beneficial effect in terms of SUDEP prevention. ${ }^{17}$ Since the precise mechanism of development of cardiac arrhythmias due to epilepsy has not yet been elucidated, it seems inappropriate to implant cardiac pacemakers and apply defibrillators in all epilepsy patients. Further studies are needed to determine which patients are at the highest risk and to determine the effectiveness of ICDs for SUDEP prevention.

Several risk factors predisposing patients to SUDEP have been considered. The severity of epilepsy, such as tonic-clonic seizures, frequent seizures, early onset epilepsy, and long duration of seizure, polytherapy with antiepileptic drugs, and nocturnal seizures have been reported as risk factors. ${ }^{1,9}$ Patients in other previous case reports had many of these risk factors. Moreover, these patients 
had a relatively long disease period with chronic, refractory, and uncontrolled seizures despite antiepileptic drug polytherapy. ${ }^{4,5}$ As for the present patient, the disease period was relatively short as the epilepsy had developed 4 years ago, and only one antiepileptic drug had been administered as epileptic seizures rarely occurred, and after administration, there had been no relapses until recently. Altogether, this was a near-SUDEP case where VF occurred during the 5th seizure since epilepsy development. Therefore, it can be inferred that SUDEP can occur early in the course of epilepsy and in patients who rarely have seizures. However, why sudden death occurs in some patients after only a few seizures is unclear. ${ }^{1}$

Convulsive syncope caused by cardiac arrhythmias is likely to be misdiagnosed as epilepsy because they share several clinical features. ${ }^{18}$ In the present patient, EEG and video-EEG monitoring were performed after several previous episodes of seizures, but the epilepsy was diagnosed based on the patient guardian's statement and treated because definite inter-ictal epileptiform discharge was absent. EEG is useful in patients with epilepsy, but its sensitivity is low. ${ }^{19}$ Video-EEG monitoring requires that seizures should occur at a sufficient frequency to be recorded over several days of examination. Therefore, EEG was not useful in the differential diagnosis of epilepsy and convulsive syncope in this case since the patient had only rare seizure events. Further examinations such as exercise treadmill testing, provocative drug ECG testing, genetic testing, and electrophysiological studies can be considered for differentiating between epileptic seizure and convulsive syncope. ${ }^{18,19}$ In this case report, the patient had a brief post-ictal period at one day before the admission; thus, convulsive syncope can be considered. However, there was a relatively long post-ictal period in the last episode, which lasted from the onset of seizures until 10 mins after arrival at the ED. Furthermore, Todd's paralysis, a reversible, temporary right limb paralysis and global aphasia, which can accompany epileptic seizures, was observed in the period between recovery of consciousness in the ED and after admission to the ICU. Thus, we believe that it is more likely that this patient's loss of consciousness and convulsive seizure were caused by an epileptic seizure rather than convulsive syncope due to primary cardiac arrhythmias, and that the VF was caused by the epileptic seizure.

As for the present patient, although the previous epileptic seizures were all nocturnal seizures that occurred during sleep, when VF occurred, the convulsive seizure occurred in an alert state during daily life activities and the duration of seizure was relatively longer when compared with the previous seizures. Moreover, the condition of the seizure changed as this episode was a tonic-clonic type seizure, whereas the previous seizures were tonic-type seizures. It is unclear whether the patient's epilepsy gradually deteriorated despite the antiepileptic drug administration.

It is unclear whether the present patient's seizurerelated VF accompanied the occurrence of seizure or if it was secondary to apnea-related hypoxia because this was a pre-hospital emergency case wherein video-EEG, ECG, and pulse oximetry were not simultaneously performed in the EMU. This is considered a limitation of the case presented here. Seizure-induced VF is likely to be underreported. This is probably because unlike in this patient, defibrillation and CPR are not performed immediately, often resulting in SUDEP. More future case reports and studies are needed to clarify the mechanisms of seizureinduced VF.

\section{Conclusion}

SUDEP is known to often occur in severely epileptic patients who have a long disease period, use multiple drugs in combination, and have a number of risk factors that cannot be well controlled. However, regardless of these factors, all patients with epilepsy are susceptible to SUDEP. When treating a patient with an epileptic seizure, one should always keep in mind the possibility of SUDEP as a seizure-induced emergency situation involving fatal arrhythmia and cardiac arrest, even in young healthy adults.

\section{Ethical statement}

Written consent had been obtained from the patient for the publication of this case report and accompanying images. Institutional approval was not required.

\section{Disclosure}

The authors report no conflicts of interest in this work.

\section{References}

1. Devinsky O. Sudden, unexpected death in epilepsy. $N$ Engl J Med. 2011;365(19):1801-1811. doi:10.1056/NEJMra1010481

2. van der Lende M, Surges R, Sander JW, Thijs RD. Cardiac arrhythmias during or after epileptic seizures. J Neurol Neurosurg Psychiatry. 2016;87 (1):69-74. doi:10.1136/jnnp-2015-310559

3. Rugg-Gunn FJ, Simister RJ, Squirrell M, Holdright DR, Duncan JS. Cardiac arrhythmias in focal epilepsy: a prospective long-term study. Lancet. 2004;364(9452):2212-2219. doi:10.1016/S0140-6736(04) 17594-6 
4. Espinosa PS, Lee JW, Tedrow UB, Bromfield EB, Dworetzky BA. Sudden unexpected near death in epilepsy: malignant arrhythmia from a partial seizure. Neurology. 2009;72(19):1702-1703. doi:10.1212/ WNL.0b013e3181a55f90

5. Ferlisi M, Tomei R, Carletti M, Moretto G, Zanoni T. Seizure induced ventricular fibrillation: a case of near-SUDEP. Seizure. 2013;22(3):249-251. doi:10.1016/j.seizure.2012.12.008

6. Shin JW, Baek IC, Oh JE, Lee JK, Jeong HS, Kim JM. Near-sudden unexpected death of epilepsy (SUDEP) caused by ventricular fibrillation following seizure in a post-stroke epilepsy patient. $J$ Korean Neurol Assoc. 2011;29(4):368-370.

7. Veiga M, Santos C, Gouveia M, Dias C. Sudden unexpected death in epilepsy and ventricular fibrillation. Resuscitation. 2015;96(Suppl 1):143. doi:10.1016/j.resuscitation.2015.09.341

8. Nashef L, So EL, Ryvlin P, Tomson T. Unifying the definitions of sudden unexpected death in epilepsy. Epilepsia. 2012;53(2):227-233. doi:10.1111/j.1528-1167.2011.03358.x

9. Double SN, Thomas SV. Sudden unexpected death in epilepsy. Indian $J$ Med Res. 2017;145(6):738-745. doi:10.4103/ijmr.IJMR 548 17

10. Stewart M. An explanation for sudden death in epilepsy (SUDEP). $J$ Physiol Sci. 2018;68(4):307-320. doi:10.1007/s12576-018-0602-z

11. Velagapudi P, Turagam M, Laurence T, Kocheril A. Cardiac arrhythmias and sudden unexpected death in epilepsy (SUDEP). Pacing Clin Electrophysiol. 2012;35(3):363-370. doi:10.1111/j.1540-8159.2011.0 3276.x

12. Lotufo PA, Valiengo L, Bensenor IM, Brunoni AR. A systematic review and meta-analysis of heart rate variability in epilepsy and antiepileptic drugs. Epilepsia. 2012;53(2):272-282. doi:10.1111/ j.1528-1167.2011.03361.x
13. Naggar I, Stewart M. A rat model for exploring the contributions of ventricular arrhythmia to sudden death in epilepsy. In: Lathers CM, Schraeder PL, Leestma JE, Wannamaker BB, Verrier RL, Schachter SC, editors. Sudden Unexpected Death in Epilepsy. Boca Raton: Taylor \& Francis, Routledge; 2015:241-250.

14. Devinsky O, Kim A, Friedman D, Bedigian A, Moffatt E, Tseng ZH. Incidence of cardiac fibrosis in SUDEP and control cases. Neurology. 2018;91(1):e55-e61. doi:10.1212/WNL.0000000000005740

15. Park KJ, Sharma G, Kennedy JD, Seyal M. Potentially high-risk cardiac arrhythmias with focal to bilateral tonic-clonic seizures and generalized tonic-clonic seizures are associated with the duration of periictal hypoxemia. Epilepsia. 2017;58(12):2164-2171. doi:10.1111/ epi.13934

16. Badheka A, Ralthod A, Kizilbash MA, et al. Epileptic patients who survived sudden cardiac death have increased risk of recurrent arrhythmias and death. J Cardiovasc Med (Hagerstown). 2010;11 (11):810-814. doi:10.2459/JCM.0b013e32833b99c1

17. Dlouhy BJ, Gehlbach BK, Richerson GB. Sudden unexpected death in epilepsy: basic mechanisms and clinical implications for prevention. J Neurol Neurosurg Psychiatry. 2016;87(4):402-413. doi:10.1136/jnnp-2013-307442

18. Burghaus L, Liu W, Eggers C, Muller-Ehmsen J, Fink GR. Mistaking a long QT syndrome for epilepsy: does every seizure call for an ECG? Fortschr Neurol Psychiatr. 2010;78(7):419-424. doi:10.1055/ s-0029-1245443

19. McKeon A, Vaughan C, Delanty N. Seizure versus syncope. Lancet Neurol. 2006;5(2):171-180. doi:10.1016/S1474-4422(06)70350-7
Open Access Emergency Medicine

\section{Publish your work in this journal}

The Open Access Emergency Medicine is an international, peerreviewed, open access journal publishing original research, reports, editorials, reviews and commentaries on all aspects of emergency medicine. The manuscript management system is completely online and includes a very quick and fair peer-review system, which is all easy to use. Visit http://www.dovepress.com/testimonials.php to read real quotes from published authors. 